\title{
Adaptive Tracking Control of Second-Order Multiagent Systems with Jointly Connected Topologies
}

\author{
Lei Chen, ${ }^{1}$ Kaiyu Qin, ${ }^{1}$ and Jiangping $\mathrm{Hu}^{2}$ \\ ${ }^{1}$ School of Aeronautics and Astronautics, University of Electronic Science and Technology of China, Chengdu 611731, China \\ ${ }^{2}$ School of Automation Engineering, University of Electronic Science and Technology of China, Chengdu 611731, China \\ Correspondence should be addressed to Lei Chen; chenleiuestc@126.com
}

Received 7 April 2016; Revised 23 June 2016; Accepted 3 July 2016

Academic Editor: Xuejun Xie

Copyright (c) 2016 Lei Chen et al. This is an open access article distributed under the Creative Commons Attribution License, which permits unrestricted use, distribution, and reproduction in any medium, provided the original work is properly cited.

\begin{abstract}
This paper considers a consensus problem of leader-following multiagent system with unknown dynamics and jointly connected topologies. The multiagent system includes a self-active leader with an unknown acceleration and a group of autonomous followers with unknown time-varying disturbances; the network topology associated with the multiagent system is time varying and not strongly connected during each time interval. By using linearly parameterized models to describe the unknown dynamics of the leader and all followers, we propose a decentralized adaptive tracking control protocol by using only the relative position measurements and analyze the stability of the tracking error and convergence of the adaptive parameter estimators with the help of Lyapunov theory. Finally, some simulation results are presented to demonstrate the proposed adaptive tracking control.
\end{abstract}

\section{Introduction}

As one kind of the major research content of distributed coordination control for multiagent systems, leader-following problem had attracted a host of researchers. For example, Ren proposed and analyzed consensus tracking algorithms in [1] and solved the leader-following problem that only a few agents can obtain a time-varying consensus reference state. Hong et al. investigated the leader-following problem, using an "observer" to solve how to track the leader with unknown velocity in [2]. Hu and Hong considered a leader-following consensus problem of a group of autonomous agents with time-varying coupling delays in [3] and investigated two different cases of coupling topologies. Peng and Yang studied the problem of multiple time-varying delays for secondorder multiagent systems in [4]. Song et al. achieved leaderfollowing consensus in a network of agents with nonlinear second-order dynamics in [5] by presenting a pinning control algorithm.

Meanwhile, estimation strategies with partial measurements and adaptive control schemes about unknown disturbances had captured some individuals' attention. Hong et al. designed the distributed observers for the second-order agents in [6], such that the velocity of the active leader cannot be measured. $\mathrm{Hu}$ et al. solved an event-triggered tracking problem in [7] by using an observer-based consensus tracking control, which is designed on the basis of a novel distributed velocity estimation technique. Zhang and Yang proposed two bounded control laws, which are independent of velocity information in [8], to deal with the finite-time consensus tracking problem. Bauso et al. considered stationary consensus protocols for networks of dynamic agents in [9] in which the neighbors' states are affected by unknown but bounded disturbances. Hu and Zheng just used the relative position measurements to design a dynamic output-feedback tracking control together with decentralized adaptive laws in [10]. Li et al. designed a distributed adaptive consensus protocol in [11] based on the agent dynamics and the relative states of neighboring agents and achieved leader-follower consensus for any communication graph which contains a directed spanning tree with the leader as the root node. Bai et al. considered the situation where the reference velocity information is available only to a leader in [12] and then developed an adaptive design to recover the desired formation.

The work about dynamically changing topologies, such as jointly connected topology, also appeared in some research. 
In [13] Hong et al. adopted a neighbor-based rule to realize local control strategies for these autonomous agents and made all the agents converge to a common value by using a Lyapunov-based approach. In [14], Lin and Jia investigated consensus problems in networks of continuous-time agents with time delays and jointly connected topologies.

In this paper, we consider a leader-following problem about second-order multiagent system, which has unknown time-varying disturbances and the system is partial measurement.

Different from some existing research, we consider the network of the system is jointly connected and prove a lemma to solve the the jointly connected topologies problem about leader-following system. This method can apply to some other jointly connected problems. Moreover, the leader's velocity and acceleration in the multiagent system are unknown; we propose a state variable to estimate the relative velocity and design a control law to guarantee the agents to follow the leader by using relative position measurement only. In addition, we propose the decentralized adaptive laws for the unknown disturbances, and with the help of a prudently chosen common Lyapunov function under a persistent excitation condition, we prove both the tracking errors and disturbances parameter estimate errors can converge to zero.

The subsequent sections are organized as follows: In Section 2, we introduce some preliminaries and present the leader-following multiagent model. In Section 3, we propose a dynamic output-feedback tracking control with two decentralized adaptive laws for each follower. Then we analyze the consensus of the system and obtain the main results in Section 4. In Section 5, we give the numerical simulation results. Finally, some conclusions are drawn in Section 6.

\section{Problem Statement}

2.1. Algebraic Graph Theory. Firstly we introduce the graph theory; we use it to describe the communication between agents in a multiagent system. Consider a tracking problem for a multiagent system about $n$ followers and one leader. The interconnection topology of $n$ followers can be conveniently described by a undirected graph $\mathscr{G}=(\mathscr{V}, E, A)$ of order $n$, where $\mathscr{V}=\left\{v_{1}, v_{2}, \ldots, v_{n}\right\}$ is the set of $n$ nodes, $E \subseteq \mathscr{V} \times \mathscr{V}$ is the set of edges, and $A=\left[a_{i j}\right]$ is a weighted adjacency matrix. The node indexes belong to a finite index set $\mathscr{I}=\{1,2, \ldots, n\}$. An edge of $\mathscr{G}$ is denoted by $e_{i j}=\left(v_{i}, v_{j}\right)$. The adjacency elements associated with the edges are positive. The adjacency matrix is defined as $a_{i i}=0$ and $a_{i j}=a_{j i} \geq 0$. When node $v_{i}$ has edge to $v_{j}, a_{i j}>0$, the vertex $j$ is called a neighbor of vertex $i$; it means that agent $j$ is communicating to agent $i$, denoted by $N_{i}$. Then $N_{i}(t)=\{j \in \mathscr{V}:(i, j) \in E, j \neq i\}$. The out-degree matrix of $\mathscr{G}$ is $D=\operatorname{diag}\left(d_{1}, \ldots, d_{n}\right) \in R^{n \times n}$, where $d_{i}=\sum_{j \in N_{i}(t)} a_{i j}$ are the diagonal elements for $i=\{1,2, \ldots, n\}$. The Laplacian of the undigraph $\mathscr{G}$ is defined as $L=D-A$.

The leader (labeled 0 ) is represented by vertex $v_{0}$, and the connection between the followers and the leader is directed. In the context of this paper, there are only parts of the followers having edges to the leader. Then, we have a simple graph
$\overline{\mathscr{G}}$ with vertex set $\overline{\mathscr{V}}=\mathscr{V} \cup\left\{v_{0}\right\}$, which contains graph $\mathscr{G}$ of $n$ followers and the leader with directed edges, if any, from some vertices of $\mathscr{G}$ to the leader vertex. Use $B$ to describe the leader adjacency matrix and $B=\operatorname{diag}\left(b_{1}, b_{2}, \ldots, b_{n}\right)$, where $b_{i}>0$ if the leader is a neighbor of agent $i$ and $b_{i}=0$ otherwise. When there is at least one directed edge from vertices of the graph $\mathscr{G}$ to the leader vertex $v_{0}$, the graph $\overline{\mathscr{G}}$ is said to be connected.

Consider an infinite sequence of nonempty, bounded, and continuous-time intervals $\left[t_{r}, t_{r+1}\right), r=0,1, \ldots$, with $t_{0}=0$, $t_{r+1}-t_{r}=T$ for some constant $T>0$. Suppose that, in each interval $\left[t_{r}, t_{r+1}\right)$, there is a sequence of nonoverlapping subintervals

$$
\begin{aligned}
{\left[t_{r_{0}}, t_{r_{1}}\right) \cdots\left[t_{r_{j}}, t_{r_{j+1}}\right) \cdots\left[t_{r_{m_{r}-1}}, t_{r_{m_{r}}}\right) } & \\
& \left(t_{r}=t_{r_{0}}, t_{r+1}=t_{r_{m_{r}}}\right)
\end{aligned}
$$

satisfying $t_{r_{1}}-t_{r_{0}}=\cdots=t_{r_{m_{r}}}-t_{r_{m_{r}-1}}=t_{\Delta}, 0 \leq j<m_{r}$, for some integer $m_{r} \geq 0$ and given constant $t_{\Delta}>0$. It is clear that there are $m_{*}=T / t_{\Delta}$ subintervals in each interval. During each of the subintervals, the interconnection topology described by $\overline{\mathscr{G}}_{m}\left(m=1, \ldots, m_{*}\right)$ is stable and changing at each time $t_{r_{j+1}}$.

The graph $\overline{\mathscr{G}}_{m}\left(m=1, \ldots, m_{*}\right)$ has the same node set $\overline{\mathscr{V}}$, and the union of the collection is defined as $\overline{\mathscr{G}}_{1-m_{*}}$, whose node set is $\overline{\mathscr{V}}$ and edge set equals the union of the edge sets of all of the graphs in the collection. However, the graph $\overline{\mathscr{G}}_{m}\left(m=1, \ldots, m_{*}\right)$ may be not strongly connected, but its union graph is connected; then we say the network is jointly connected.

Lemma 1 (Godsil and Royle [15]). If the graph $G$ is connected, its Laplacian L satisfies the following: (1) zero is a simple eigenvalue of $L$, and $1_{n}$ is the corresponding eigenvector; (2) the remaining $n-1$ eigenvalues are all positive and real.

Lemma 2 (Hong et al. [2]). Denote $H=L+B$, where $L$ is the weighted Laplacian of graph $\mathscr{G}$ and $B$ is the leader adjacency matrix as defined in Section 2. If graph $\mathscr{G}$ is connected, then the symmetric matrix $H$ associated with $\overline{\mathscr{G}}$ is positive definite. Moreover, matrices $H_{1}, \ldots, H_{m_{*}}$ are associated with the graphs $\overline{\mathscr{G}}_{1}, \overline{\mathscr{G}}_{2}, \ldots, \overline{\mathscr{G}}_{m_{*}}$, respectively; $H_{i}$ is positive semidefinite because both $L_{i}$ and $B_{i}$ are positive semidefinite.

Lemma 3 (Hong et al. [13] and Lin et al. [16]). Graph $\overline{\mathscr{G}}$ is jointly connected and has $l_{\sigma} \geq 1$ connected topology in each subinterval. The corresponding nodes sets of the connected components are denoted by $\varepsilon_{r_{j}}^{1}, \varepsilon_{r_{j}}^{2}, \ldots, \varepsilon_{r_{j}}^{l_{\sigma}}$, $d_{\sigma}^{\epsilon}$ denotes the number of nodes in $\varepsilon_{r_{j}}^{\epsilon}$, and $\sum_{\epsilon=1}^{l_{\sigma}} d_{\sigma}^{\epsilon}=n$. From some knowledge of matrix theory, there exists a permutation matrix $E_{\sigma} \in R^{n \times n}$, which satisfies

$$
E_{\sigma}{ }^{T} H_{\sigma} E_{\sigma}=\operatorname{diag}\left[H_{\sigma}{ }^{1}, H_{\sigma}{ }^{2}, \ldots, H_{\sigma}{ }^{l_{\sigma}}\right] .
$$


2.2. Problem Statement. In this paper, the dynamics of each agent is described by

$$
\begin{aligned}
& \dot{x}_{i}(t)=v_{i}(t), \\
& \dot{v}_{i}(t)=u_{i}(t)+f\left(x_{i}, t\right),
\end{aligned}
$$

$$
i=1,2, \ldots, n,
$$

where $x_{i}, v_{i}, u_{i} \subset R, x_{i}, v_{i}$ are the position and velocity vectors of the $i$ th agent, respectively, and $u_{i}$ is the control input.

$f\left(x_{i}, t\right)$ is the dynamics of agent $i$, which is assumed to be an unknown time-varying disturbance.

The dynamics of the leader in the multiagent system is described by

$$
\begin{aligned}
& \dot{x}_{0}(t)=v_{0}(t), \\
& \dot{v}_{0}(t)=a_{0}(t),
\end{aligned}
$$

where $a_{0}(t)$ is an unknown acceleration of the leader.

Our aim is to design a decentralized control scheme for each agent and study under what conditions the agents can follow the leader (i.e., $\lim _{t \rightarrow \infty}\left(x_{i}(t)-x_{0}(t)\right)=0$, $\left.\lim _{t \rightarrow \infty}\left(v_{i}(t)-v_{0}(t)\right)=0\right)$.

\section{Adaptive Control Design}

Before giving the adaptive control law, we propose two variables to estimate $a_{0}(t)$ and $f\left(x_{i}, t\right)$.

For leader-follower system, the acceleration $a_{0}(t)$ and the disturbances $f\left(x_{i}, t\right)(i=1, \ldots, n)$ are unknown. By the techniques in classical adaptive control (Marino and Tomei [17]) and multiagent systems (Bai et al. [12], Hu and Zheng $[10])$, they can be parameterized, respectively, as follows:

$$
\begin{aligned}
a_{0}(t) & =\phi_{o}(t) \omega_{o}(t), \\
f\left(x_{i}, t\right) & =\phi_{i}(t) \omega_{i}(t),
\end{aligned}
$$

where $\phi_{o}(t), \phi_{i}(t) \in R_{m}$ are basis function vectors and $\omega_{o}, \omega_{i} \in$ $R_{m}$ are unknown constant parameter vectors that will be estimated.

Each follower $i$ estimates the parameter vectors $\omega_{o}, \omega_{i}$ by $\widetilde{\omega}_{o i}(t), \widetilde{\omega}_{i}(t) \in R_{m}$ and estimates $a_{0}(t), f\left(x_{i}, t\right)$ by $a_{i}(t)$, and $\widetilde{f}\left(x_{i}, t\right)$, respectively. So we have

$$
\begin{aligned}
a_{i}(t) & =\phi_{o}(t) \widetilde{\omega}_{o i}(t), \\
\widetilde{f}\left(x_{i}, t\right) & =\phi_{i} \widetilde{\omega}_{i}(t),
\end{aligned}
$$

for $i=1, \ldots, n$.

Secondly, we define two variables to describe the relative measurement of position and velocity.

The relative position measurement is

$$
\theta_{i}(t)=\sum_{j \in N_{i}(t)} a_{i j}\left(x_{i}(t)-x_{j}(t)\right)+b_{i}\left(x_{i}(t)-x_{0}(t)\right),
$$

for $i=1, \ldots, n$.
The relative velocity measurement is

$$
\vartheta_{i}(t)=\sum_{j \in N_{i}(t)} a_{i j}\left(v_{i}(t)-v_{j}(t)\right)+b_{i}\left(v_{i}(t)-v_{0}(t)\right),
$$

for $i=1, \ldots, n$.

So from the above definition, the following statements are equivalent:

(1) $\lim _{t \rightarrow \infty} \theta=0$ and $\lim _{t \rightarrow \infty} \vartheta=0$.

(2) $\lim _{t \rightarrow \infty}\left(x-x_{0}\right)=0$ and $\lim _{t \rightarrow \infty}\left(v-v_{0}\right)=0$. $\vartheta_{i}(t)$

Differentiating the two relative measurements $\theta_{i}(t)$ and

$$
\begin{aligned}
\dot{\theta}_{i}(t)= & \vartheta_{i}(t), \\
\dot{\vartheta}_{i}(t)= & \sum_{j \in N_{i}(t)} a_{i j}\left(u_{i}(t)-u_{j}(t)+f\left(x_{i}, t\right)-f\left(x_{j}, t\right)\right) \\
& +b_{i}\left(u_{i}(t)+f\left(x_{i}, t\right)-a_{0}(t)\right) .
\end{aligned}
$$

Thus, we take

$$
\begin{aligned}
\bar{x}_{i}(t) & =x_{i}(t)-x_{0}(t), \\
\bar{v}_{i}(t) & =v_{i}(t)-v_{0}(t), \\
\bar{x} & =\operatorname{col}\left\{\bar{x}_{1}(t), \bar{x}_{2}(t), \ldots, \bar{x}_{n}(t)\right\}, \\
\bar{v} & =\operatorname{col}\left\{\bar{v}_{1}(t), \bar{v}_{2}(t), \ldots, \bar{v}_{n}(t)\right\}, \\
\theta(t) & =\operatorname{col}\left\{\theta_{1}(t), \theta_{2}(t), \ldots, \theta_{n}(t)\right\}, \\
\vartheta(t) & =\operatorname{col}\left\{\vartheta_{1}(t), \vartheta_{2}(t), \ldots, \vartheta_{n}(t)\right\} .
\end{aligned}
$$

Then the system can be simplified as

$$
\begin{aligned}
& \theta(t)=H_{\sigma} \bar{x}(t), \\
& \vartheta(t)=H_{\sigma} \bar{v}(t) .
\end{aligned}
$$

Furthermore,

$$
\begin{aligned}
\dot{\theta}(t) & =\vartheta(t) \\
\dot{\vartheta}(t) & =H_{\sigma}\left[u(t)-a_{0}(t) \mathbf{1}_{n}+f(x, t)\right], \\
f(x, t) & =\left(\begin{array}{c}
f\left(x_{1}, t\right) \\
f\left(x_{2}, t\right) \\
\vdots \\
f\left(x_{n}, t\right)
\end{array}\right), \\
u & =\left(\begin{array}{c}
u_{1} \\
u_{2} \\
\vdots \\
u_{n}
\end{array}\right) .
\end{aligned}
$$

$\mathbf{1}_{n}$ denote a column vector where all the elements are 1. 
$\sigma:[0, \infty) \rightarrow \mathscr{P}\left\{1,2, \ldots, m_{*}\right\} \quad\left(m_{*}\right.$ denotes the total number of all possible graphs) is a switching signal that determines the communication topology $\overline{\mathscr{G}}$.

$L$ is the Laplacian for the $n$ followers; the leader adjacency matrix $B$ is an $n \times n$ diagonal matrix whose $i$ th diagonal element is $b_{i}(t)$ at time $t$ and is utilized to represent the connections between the followers and the leader.

Now, we consider the control protocol.

If $v_{0}, \omega_{o}$, and $\omega_{i}$ are known, we can design the control protocol as

$$
u_{i}(t)=\phi_{o}(t) \omega_{o}-\gamma \theta_{i}(t)-k \vartheta_{i}(t)-\phi_{i}(t) \omega_{i},
$$

but in our cases, $v_{0}, \omega_{o}$, and $\omega_{i}$ are unknown; we define $\eta_{i}(t)$ as the estimate of $\vartheta_{i}(t)$ by agent $i$. Then the control protocol is

$$
u_{i}(t)=\phi_{o}(t) \widetilde{\omega}_{o i}(t)-\gamma \theta_{i}(t)-k \eta_{i}(t)-\phi_{i}(t) \widetilde{\omega}_{i}(t) .
$$

From (14), $\eta_{i}(t)$ is unknown, so we design the parameter input of $\eta_{i}(t)$ as

$$
\begin{aligned}
\dot{\eta}_{i}(t)=-l\left(\eta_{i}(t)-\vartheta_{i}(t)\right) & \\
- & \gamma\left[\sum_{j \in N_{i}(t)} a_{i j}\left(\theta_{i}(t)-\theta_{j}(t)\right)+b_{i} \theta_{i}(t)\right] \\
& -k\left[\sum_{j \in N_{i}(t)} a_{i j}\left(\eta_{i}(t)-\eta_{j}(t)\right)+b_{i} \eta_{i}(t)\right], \\
l>1 . &
\end{aligned}
$$

Lemma 4. When (15) is satisfied, without consideration of the parameter error of $\omega(t)_{0}$ and $\omega(t)_{i}, \eta_{i}(t)$ is the estimate of $\mathcal{\vartheta}_{i}(t)$; that is, $\lim _{t \rightarrow \infty}\left(\eta_{i}(t)-\vartheta_{i}(t)\right)=0$.

Proof. From (14) and (15),

$$
\begin{aligned}
\dot{\eta}_{i}(t) & \\
= & -l\left(\eta_{i}(t)-\vartheta_{i}(t)\right) \\
& +\sum_{j \in N_{i}(t)} a_{i j}\left(u_{i}(t)-u_{j}(t)+f\left(x_{i}, t\right)-f\left(x_{j}, t\right)\right) \\
& +b_{i}\left(u_{i}(t)+f\left(x_{i}, t\right)-a_{0}(t)\right) .
\end{aligned}
$$

When $\widetilde{\omega}_{0 i}(t)=\omega_{0}(t)$ and $\widetilde{\omega}_{i}(t)=\omega_{i}(t)$, from $(9)$

$$
\begin{aligned}
\dot{\eta}_{i}(t) & =-l\left(\eta_{i}(t)-\vartheta_{i}(t)\right)+\dot{\vartheta}_{i}(t), \\
\dot{\eta}_{i}(t)-\dot{\vartheta}_{i}(t) & =-l\left(\eta_{i}(t)-\vartheta_{i}(t)\right) .
\end{aligned}
$$

It is equal to

$$
\frac{d}{d t}\left(\eta_{i}(t)-\vartheta_{i}(t)\right)=-l\left(\eta_{i}(t)-\vartheta_{i}(t)\right) .
$$

That is, $\lim _{t \rightarrow \infty}\left(\eta_{i}(t)-\vartheta_{i}(t)\right)=0$.

This completes the proof.
In our case, $\vartheta_{i}(t)$ is unknown, so we define a variable $\widehat{\eta}_{i}$ for each agent $i$ and set

$$
\widehat{\eta}_{i}=\eta_{i}-l \theta_{i}
$$

and then (15) can be rewritten as

$$
\begin{aligned}
\dot{\vec{\eta}}_{i}(t) & \\
= & -(\gamma+l k)\left[\sum_{j \in N_{i}(t)} a_{i j}\left(\theta_{i}(t)-\theta_{j}(t)\right)+b_{i} \theta_{i}(t)\right] \\
& -k\left[\sum_{j \in N_{i}(t)} a_{i j}\left(\hat{\eta}_{i}(t)-\hat{\eta}_{j}(t)\right)+b_{i} \widehat{\eta}_{i}(t)\right] \\
& -l \hat{\eta}_{i}(t)-l^{2} \theta_{i}(t) .
\end{aligned}
$$

From (19) and (20), we can use only related position measurement to estimate the relative velocity measurement, so the tracking control is

$$
\begin{aligned}
& \dot{\hat{\eta}}_{i}(t) \\
& =-(\gamma+l k)\left[\sum_{j \in N_{i}(t)} a_{i j}\left(\theta_{i}(t)-\theta_{j}(t)\right)+b_{i} \theta_{i}(t)\right] \\
& \quad-k\left[\sum_{j \in N_{i}(t)} a_{i j}\left(\widehat{\eta}_{i}(t)-\widehat{\eta}_{j}(t)\right)+b_{i} \widehat{\eta}_{i}(t)\right] \\
& \quad-l \widehat{\eta}_{i}(t)-l^{2} \theta_{i}(t), \\
& u_{i}(t) \quad \\
& =-(\gamma+k l) \theta_{i}(t)-k \widehat{\eta}_{i}(t)-\phi_{i} \widetilde{\omega}_{i}(t) \\
& \quad+\phi_{o}(t) \widetilde{\omega}_{o i}(t) .
\end{aligned}
$$

And equality is

$$
\begin{aligned}
\dot{\hat{\eta}}(t)= & -\left(l^{2} I_{n}+l k H_{\sigma}+\gamma H_{\sigma}\right) \theta(t) \\
& -\left(k H_{\sigma}+l I_{n}\right) \hat{\eta}(t), \\
u(t)= & -(\gamma+k l) \theta(t)-k \widehat{\eta}(t)+\Phi_{o}(t) \widetilde{\Omega}_{o}(t) \\
& -\Phi \widetilde{\Omega}(t),
\end{aligned}
$$

where

$$
\begin{aligned}
\widehat{\eta} & =\operatorname{col}\left\{\widehat{\eta}_{1}, \widehat{\eta}_{2}, \ldots, \widehat{\eta}_{n}\right\}, \\
\Phi_{o}(t) & =\operatorname{diag}\left\{\phi_{0}, \ldots, \phi_{0}\right\}, \\
\Phi(t) & =\operatorname{diag}\left\{\phi_{1}, \ldots, \phi_{n}\right\}, \\
\widetilde{\Omega}_{0} & =\operatorname{col}\left\{\widetilde{\omega}_{01}, \ldots, \widetilde{\omega}_{0 n}\right\}, \\
\widetilde{\Omega}_{0} & =\operatorname{col}\left\{\widetilde{\omega}_{1}, \ldots, \widetilde{\omega}_{n}\right\} .
\end{aligned}
$$


Thus we have designed the control protocol only using the relative position measurement, and it is similar to the protocol, which Hu and Zheng designed in [10].

Now we design the adaptive laws. Firstly we define two parameter variables $\widehat{\omega}_{i 0}(t)$ and $\widehat{\omega}_{i}(t)$ and let

$$
\begin{aligned}
\widehat{\omega}_{0 i}(t)= & \widetilde{\omega}_{0 i}(t) \\
& +2 \phi_{0}\left[\sum_{j \in N_{i}(t)} a_{i j}\left(\theta_{i}(t)-\theta_{j}(t)\right)+b_{i} \theta_{i}(t)\right], \\
\widehat{\omega}_{i}(t)= & \widetilde{\omega}_{i}(t) \\
& -2 \phi_{i}\left[\sum_{j \in N_{i}(t)} a_{i j}\left(\theta_{i}(t)-\theta_{j}(t)\right)+b_{i} \theta_{i}(t)\right] .
\end{aligned}
$$

Then

$$
\begin{aligned}
\lim _{t \rightarrow \infty} \widehat{\omega}_{0 i}(t) & =\widetilde{\omega}_{0 i}(t), \\
\lim _{t \rightarrow \infty} \widehat{\omega}_{i}(t) & =\widetilde{\omega}_{i}(t) .
\end{aligned}
$$

Consider $\lim _{t \rightarrow \infty} \theta_{i}(t)=0$. That is to say, we can design the adaptive laws for $\widehat{\omega}_{0 i}(t)$ and $\widehat{\omega}_{i}(t)$ to get the value of $\widetilde{\omega}_{0 i}(t)$ and $\widetilde{\omega}_{i}(t)$.

We design the adaptive laws for the two variables $\widehat{\omega}_{0 i}(t)$ and $\widehat{\omega}_{i}(t)$ as

$$
\begin{aligned}
\dot{\hat{\omega}}_{0 i}(t) & =\left(l \phi_{0}-\phi_{0}+2 \dot{\phi}_{0}\right) \\
\cdot & {\left[\sum_{j \in N_{i}(t)} a_{i j}\left(\theta_{i}(t)-\theta_{j}(t)\right)+b_{i} \theta_{i}(t)\right] } \\
+ & \phi_{0}\left[\sum_{j \in N_{i}(t)} a_{i j}\left(\hat{\eta}_{i}(t)-\widehat{\eta}_{j}(t)\right)+b_{i} \widehat{\eta}_{i}(t)\right], \\
\dot{\hat{\omega}}_{i}(t) & =-\left(l \phi_{i}-\phi_{i}+2 \dot{\phi}_{i}\right) \\
\cdot & {\left[\sum_{j \in N_{i}(t)} a_{i j}\left(\theta_{i}(t)-\theta_{j}(t)\right)+b_{i} \theta_{i}(t)\right] } \\
- & {\left[\phi_{i}\left[\sum_{j \in N_{i}(t)} a_{i j}\left(\widehat{\eta}_{i}(t)-\widehat{\eta}_{j}(t)\right)+b_{i} \widehat{\eta}_{i}(t)\right] .\right.}
\end{aligned}
$$

Equation (26) can be rewritten as

$$
\begin{aligned}
\dot{\widehat{\Omega}}_{0}(t)= & \left(l \Phi_{0}(t)-\Phi_{0}(t)+2 \dot{\Phi}_{0}(t)\right) H_{\sigma} \theta(t) \\
& +\Phi_{0} H_{\sigma} \hat{\eta}(t), \\
\dot{\widehat{\Omega}}(t)= & -(l \Phi(t)-\Phi(t)+2 \dot{\Phi}(t)) H_{\sigma} \theta(t) \\
& -\Phi H_{\sigma} \hat{\eta}(t),
\end{aligned}
$$

$$
\begin{aligned}
\widehat{\Omega}_{0}(t) & =\operatorname{col}\left\{\widehat{\Omega}_{01}(t), \ldots, \widehat{\Omega}_{0 n}(t)\right\}, \\
\widehat{\Omega}(t) & =\operatorname{col}\left\{\widehat{\Omega}_{1}(t), \ldots, \widehat{\Omega}_{n}(t)\right\}, \\
\Phi_{0}(t) & =I_{n} \otimes \phi_{0}(t), \\
\Phi(t) & =\operatorname{diag}\left\{\phi_{1}(t), \ldots, \phi_{n}(t)\right\} .
\end{aligned}
$$

Set

$$
\begin{aligned}
\bar{\Omega}_{0}(t) & =\operatorname{col}\left\{\widetilde{\omega}_{01}(t)-\omega_{0}(t), \ldots, \widetilde{\omega}_{0 n}(t)-\omega_{0}(t)\right\}, \\
\bar{\Omega}(t) & =\operatorname{col}\left\{\widetilde{\omega}_{1}(t)-\omega_{1}(t), \ldots, \widetilde{\omega}_{n}(t)-\omega_{n}(t)\right\} .
\end{aligned}
$$

Then we know $\dot{\bar{\Omega}}_{0}(t)=\dot{\widetilde{\Omega}}_{0}(t)$ and $\dot{\bar{\Omega}}(t)=\dot{\widetilde{\Omega}}(t)$, and adaptive laws (27) can be transformed to

$$
\begin{aligned}
\dot{\bar{\Omega}}_{0}(t)= & -\Phi_{0}(t) H_{\sigma} \theta(t)-2 \Phi_{0}(t) H_{\sigma} \vartheta(t) \\
& +\Phi_{0}(t) H_{\sigma} \eta(t), \\
\dot{\bar{\Omega}}(t)= & \Phi(t) H_{\sigma} \theta(t)+2 \Phi(t) H_{\sigma} \vartheta(t) \\
& -\Phi(t) H_{\sigma} \eta(t) .
\end{aligned}
$$

\section{Consensus Analysis}

From the above design and definition, we can rewrite the system as

$$
\begin{aligned}
\dot{\theta}(t)= & \mathcal{\vartheta}(t), \\
\dot{\vartheta}(t)= & -\gamma H_{\sigma} \theta(t)-k H_{\sigma} \eta(t)+H_{\sigma} \Phi_{0} \bar{\Omega}_{0}(t) \\
& -H_{\sigma} \Phi \bar{\Omega}(t), \\
\dot{\eta}(t)= & l \vartheta(t)-\gamma H_{\sigma} \theta(t)-\left(l+k H_{\sigma}\right) \eta(t),
\end{aligned}
$$

which is equal to

$$
\begin{aligned}
\dot{\delta} & =F_{\sigma} \delta(t)+\Delta(t), \\
\delta & =\left[\begin{array}{l}
\theta \\
\vartheta \\
\eta
\end{array}\right] \\
F_{\sigma} & =\left[\begin{array}{ccc}
0 & I_{n} & 0 \\
-\gamma H_{\sigma} & 0 & -k H_{\sigma} \\
-\gamma H_{\sigma} & l I_{n} & -\left(l I_{n}+k H_{\sigma}\right)
\end{array}\right], \\
\Delta(t) & =\left[\begin{array}{cc}
H_{\sigma} \Phi_{0}(t) \bar{\Omega}_{0}(t)-H_{\sigma} \Phi(t) \bar{\Omega}(t) \\
0
\end{array}\right] .
\end{aligned}
$$

That is, when $\lim _{t \rightarrow \infty} \delta(t)=0$, then $\lim _{t \rightarrow \infty} \bar{x}(t)=0$ and $\lim _{t \rightarrow \infty} \bar{v}(t)=0$. 
By using the definitions and properties of jointly connected topology, we have

$$
\begin{aligned}
& \bar{x}^{T}(t) E_{\sigma}=\left[\bar{x}_{\sigma}^{1^{T}}(t), \bar{x}_{\sigma}^{2^{T}}(t), \ldots, \bar{x}_{\sigma}^{l^{T}}(t)\right], \\
& \bar{v}^{T}(t) E_{\sigma}=\left[\bar{v}_{\sigma}^{T^{T}}(t), \bar{v}_{\sigma}^{2^{T}}(t), \ldots, \bar{v}_{\sigma}^{l_{\sigma}^{T}}(t)\right], \\
& \eta^{T}(t) E_{\sigma}=\left[\eta_{\sigma}^{1^{T}}(t), \eta_{\sigma}^{2^{T}}(t), \ldots, \eta_{\sigma}^{l_{\sigma}^{T}}(t)\right] .
\end{aligned}
$$
get

The matrix $E_{\sigma}$ is a permutation matrix, $E_{\sigma}^{T} E_{\sigma}=I_{n}$, so we

$$
\begin{aligned}
\theta^{T}(t) E_{\sigma} & =\bar{x}^{T}(t) E_{\sigma} E_{\sigma}^{T} H^{T} E_{\sigma} \\
& =\left[\theta_{\sigma}^{1^{T}}(t), \theta_{\sigma}^{2^{T}}(t), \ldots, \theta_{\sigma}^{l_{\sigma}^{T}}(t)\right], \\
\vartheta^{T}(t) E_{\sigma} & =\bar{v}^{T}(t) E_{\sigma} E_{\sigma}^{T^{T}} H^{T} E_{\sigma} \\
& =\left[\vartheta_{\sigma}^{1^{T}}(t), \vartheta_{\sigma}^{2^{T}}(t), \ldots, \vartheta_{\sigma}^{l_{\sigma}^{T}}(t)\right] .
\end{aligned}
$$

Meanwhile,

$$
\begin{aligned}
& E_{\sigma}^{T} H_{\sigma} \Phi_{0}(t) \bar{\Omega}_{0}(t)=E_{\sigma}^{T} H_{\sigma} E_{\sigma} E_{\sigma}^{T} \Phi_{0}(t) \bar{\Omega}_{0}(t), \\
& E_{\sigma}^{T} H_{\sigma} \Phi(t) \bar{\Omega}(t)=E_{\sigma}^{T} H_{\sigma} E_{\sigma} E_{\sigma}^{T} \Phi(t) \bar{\Omega}(t), \\
& E_{\sigma}^{T} \Phi_{0}(t) \bar{\Omega}_{0}(t) \\
& \quad=\left[\Phi_{0_{\sigma}}^{1}(t) \bar{\Omega}_{0_{\sigma}}^{1}(t), \ldots, \Phi_{0_{\sigma}}^{l_{\sigma}}(t) \bar{\Omega}_{0_{\sigma}}^{l_{\sigma}^{T}}(t)\right]^{T} \\
& E_{\sigma}^{T} \Phi(t) \bar{\Omega}(t)=\left[\Phi_{\sigma}^{1}(t) \bar{\Omega}_{\sigma}^{1}(t), \ldots, \Phi_{\sigma}^{l_{\sigma}}(t) \bar{\Omega}_{\sigma}^{l_{\sigma}}(t)\right]^{T},
\end{aligned}
$$

so

$$
\begin{aligned}
& E_{\sigma}^{T} H_{\sigma} \Phi_{0}(t) \bar{\Omega}_{0}(t) \\
& \quad=\left[H_{\sigma}{ }^{1} \Phi_{0_{\sigma}}^{1}(t) \bar{\Omega}_{0_{\sigma}}^{1}(t), \ldots, H_{\sigma}{ }^{l_{\sigma}} \Phi_{0_{\sigma}}^{l_{\sigma}}(t) \bar{\Omega}_{0_{\sigma}}^{l_{\sigma}{ }^{T}}(t)\right]^{T}, \\
& E_{\sigma}^{T} H_{\sigma} \Phi(t) \bar{\Omega}(t) \\
& \quad=\left[H_{\sigma}{ }^{1} \Phi_{\sigma}^{1}(t) \bar{\Omega}_{\sigma}^{1}(t), \ldots, H_{\sigma}^{l_{\sigma}} \Phi_{\sigma}^{l_{\sigma}}(t) \bar{\Omega}_{\sigma}^{l_{\sigma}}(t)\right]^{T},
\end{aligned}
$$

where

$$
\epsilon=1, \ldots, l_{\sigma},
$$

$L_{\sigma}{ }^{\epsilon} \in R^{d_{\sigma}^{e} \times d_{\sigma}^{e}}$ is the Laplacian of the corresponding connected graph,

$B_{\sigma}{ }^{\epsilon} \in R^{d_{\sigma}^{\epsilon} \times d_{\sigma}^{\epsilon}}$ is the leader adjacency matrix of each connected graph.

Thus $H_{\sigma}^{\epsilon}=L_{\sigma}^{\epsilon}+B_{\sigma}^{\epsilon}$.

Each block matrix $H_{\sigma}^{\epsilon}$ describes the connection of the corresponding connected components.
According to the discussion above, in each subinterval, the control scheme of each connected component is

$$
\begin{aligned}
\dot{\delta}_{\sigma}^{\epsilon}(t) & =F_{\sigma}^{\epsilon} \delta_{\sigma}^{\epsilon}(t)+\Delta_{\sigma}^{\epsilon}(t), \\
\delta_{\sigma}^{\epsilon} & =\left[\begin{array}{c}
\theta_{\sigma}^{\epsilon} \\
\vartheta_{\sigma}^{\epsilon} \\
\eta_{\sigma}^{\epsilon}
\end{array}\right],
\end{aligned}
$$

$$
\begin{aligned}
F_{\sigma}^{\epsilon} & =\left[\begin{array}{ccc}
0 & I_{d_{\sigma}^{\epsilon}} & 0 \\
-\gamma H_{\sigma}^{\epsilon} & 0 & -k H_{\sigma}^{\epsilon} \\
-\gamma H_{\sigma}^{\epsilon} & l I_{d_{\sigma}^{\epsilon}} & -\left(l I_{d_{\sigma}^{\epsilon}}+k H_{\sigma}^{\epsilon}\right)
\end{array}\right], \\
\Delta_{\sigma}^{\epsilon}(t) & =\left[\begin{array}{c}
0 \\
H_{\sigma}^{\epsilon} \Phi_{0_{\sigma}}^{\epsilon}(t) \bar{\Omega}_{0_{\sigma}}^{\epsilon}(t)-H_{\sigma}^{\epsilon} \Phi_{\sigma}^{\epsilon}(t) \bar{\Omega}_{\sigma}^{\epsilon}(t) \\
0
\end{array}\right] .
\end{aligned}
$$

Lemma 5. When graphs $\overline{\mathscr{G}}_{\sigma}$ are jointly connected, the leader connects to one follower at least; then

$$
\begin{aligned}
\underline{\lambda}_{H_{\sigma}^{e}} & \geq 0, \\
\sum_{\epsilon=1}^{l_{\sigma}} \underline{\lambda}_{H_{\sigma}^{e}} & >0,
\end{aligned}
$$

where $\underline{\lambda}_{H_{\sigma}^{e}}$ is the minimum eigenvalue of $H_{\sigma}^{\epsilon}\left(\epsilon=1, \ldots, l_{\sigma}\right)$.

Proof. Assuming the leader is connected to one follower in the $\chi$ th connected components, thus $B_{\sigma}^{\chi}>0$ and $B_{\sigma}^{\epsilon}=0(\epsilon \neq$ $\chi)$; then $L_{\sigma}{ }^{\epsilon}=H_{\sigma}{ }^{\epsilon}(\epsilon \neq \chi)$, and

$$
E_{\sigma}{ }^{T} H_{\sigma} E_{\sigma}=\operatorname{diag}\left[L_{\sigma}{ }^{1}, L_{\sigma}{ }^{2}, \ldots, H_{\sigma}{ }^{\chi}, \ldots, L_{\sigma}^{l_{\sigma}}\right]
$$

According to Lemmas 1 and $2, \underline{\lambda}_{H_{\sigma}^{\chi}}>0$ and when $i \neq \chi$, $\underline{\lambda}_{H_{\sigma}^{e}}=0$. So $\sum_{\epsilon=1}^{l_{\sigma}} \underline{\lambda}_{H_{\sigma}^{e}}>0$.

The proof is complete.

Lemma 6. Consider a function $f(\delta)=\delta^{T} \Upsilon \delta$, when $\Upsilon=$ $\left[\begin{array}{lll}b_{1} & a_{1} & a_{2} \\ a_{1} & b_{2} & a_{3} \\ a_{2} & a_{3} & b_{3}\end{array}\right] \otimes I_{n}$, and $a_{1}, a_{2}, a_{3}, b_{1}, b_{2}, b_{3}$ are constants; then

$$
\begin{aligned}
\delta^{T} \Upsilon \delta & =\sum_{\epsilon=1}^{l_{\sigma}} \delta_{\sigma}^{\epsilon^{T}} \Upsilon_{\sigma}^{\epsilon} \delta_{\sigma}^{\epsilon}, \\
\Upsilon_{\sigma}^{\epsilon} & =\left[\begin{array}{lll}
b_{1} & a_{1} & a_{2} \\
a_{1} & b_{2} & a_{3} \\
a_{2} & a_{3} & b_{3}
\end{array}\right] \otimes I_{d_{\sigma}^{\epsilon}} .
\end{aligned}
$$


Proof. From Lemma 3 and $E_{\sigma}^{T} E_{\sigma}=I_{n}$, thus

$$
\begin{aligned}
\delta^{T} \Upsilon \delta= & \delta^{T}\left[\begin{array}{ccc}
E_{\sigma} & 0 & 0 \\
0 & E_{\sigma} & 0 \\
0 & 0 & E_{\sigma}
\end{array}\right]\left[\begin{array}{ccc}
E_{\sigma}^{T} & 0 & 0 \\
0 & E_{\sigma}^{T} & 0 \\
0 & 0 & E_{\sigma}^{T}
\end{array}\right] \Upsilon \\
& \times\left[\begin{array}{ccc}
E_{\sigma} & 0 & 0 \\
0 & E_{\sigma} & 0 \\
0 & 0 & E_{\sigma}
\end{array}\right]\left[\begin{array}{ccc}
E_{\sigma}^{T} & 0 & 0 \\
0 & E_{\sigma}^{T} & 0 \\
0 & 0 & E_{\sigma}^{T}
\end{array}\right] \delta \\
= & \theta^{T} E_{\sigma} E_{\sigma}^{T} \Upsilon E_{\sigma} E_{\sigma}^{T} \theta+\vartheta^{T} E_{\sigma} E_{\sigma}^{T} \Upsilon E_{\sigma} E_{\sigma}^{T} \vartheta \\
& +\eta^{T} E_{\sigma} E_{\sigma}^{T} \Upsilon E_{\sigma} E_{\sigma}^{T} \eta \\
= & \theta_{\sigma}^{1^{T}} \Upsilon_{\sigma}^{1^{T}} \theta_{\sigma}^{1}+\cdots+\theta_{\sigma}^{l^{T}} \Upsilon_{\sigma}^{l_{\sigma}^{T}} \theta_{\sigma}^{l_{\sigma}}+\vartheta_{\sigma}^{1^{T}} \Upsilon_{\sigma}^{1^{T}} \vartheta_{\sigma}^{1}+\cdots \\
& +\vartheta_{\sigma}^{l_{\sigma}^{T}} \Upsilon_{\sigma}^{l^{T}} \vartheta_{\sigma}^{l_{\sigma}}+\eta_{\sigma}^{1^{T}} \Upsilon_{\sigma}^{1^{T}} \eta_{\sigma}^{1}+\cdots+\eta_{\sigma}^{l_{\sigma}^{T}} \Upsilon_{\sigma}^{l_{\sigma}^{T}} \eta_{\sigma}^{l_{\sigma}} \\
= & \sum_{\epsilon=1}^{l_{\sigma}} \delta_{\sigma}^{\epsilon^{T}} \Upsilon_{\sigma}^{\epsilon} \delta_{\sigma}^{\epsilon} .
\end{aligned}
$$

The proof is complete.

Lemma 7 (Barbalat's lemma, Popov [18]). If a function $f(t)$ is uniformly continuous and $\lim _{t \rightarrow 0} \int_{0}^{t} f(s) d$ s exists and is finite, then $\lim _{t \rightarrow 0} f(t)=0$.

Theorem 8. Consider the leader-follower system (32). The interconnection network of the system is jointly connected across each time interval $\left[t_{r}, t_{r+1}\right)(r=0,1, \ldots)$ and $\phi_{i},(i=$ $0,1, \ldots, n)$ are uniformly bounded. When $k$ and $\gamma$ satisfy (44), the consensus tracking is achieved:

$$
\begin{aligned}
k<2 \sqrt{\frac{4 l \gamma}{\bar{\lambda}_{H_{\sigma}^{e}}^{e}}} & \sqrt{16 l\left(\bar{\lambda}_{H_{\sigma}^{e}}^{2}-1\right)\left(k^{2} \bar{\lambda}_{H_{\sigma}^{e}}^{4}(l-1)\right)+M^{2}}-M \\
\gamma> & \frac{8 l\left(\bar{\lambda}_{H_{\sigma}^{e}}^{2}+1\right)}{M}=\bar{\lambda}_{H_{\sigma}^{\epsilon}\left[3 k^{2}-4 l^{2}-8 k l+\bar{\lambda}_{H_{\sigma}^{e}}\left(4 l^{2}-l+k^{2}+8 k l\right)\right.} \\
& \left.-\frac{9}{4} \bar{\lambda}_{H_{\sigma}^{e}}^{2} k^{2}\right],
\end{aligned}
$$

where $\bar{\lambda}_{H_{\sigma}^{e}}^{2}$ is the maximal eigenvalue of $H_{\sigma}^{\epsilon}$.

Proof. Consider a common Lyapunov function candidate

$$
\begin{aligned}
V(t) & =\delta(t)^{T} P \delta(t)+\bar{\Omega}_{0}^{T}(t) \bar{\Omega}_{0}(t)+\bar{\Omega}^{T}(t) \bar{\Omega}(t), \\
P & =\left[\begin{array}{ccc}
2 \gamma I_{n} & I_{n} & 0 \\
I_{n} & 2 I_{n} & -I_{n} \\
0 & -I_{n} & I_{n}
\end{array}\right]>0, \quad \gamma>1 .
\end{aligned}
$$

Then the derivative of $V(t)$ along the trajectory of system (32) is given by

$$
\begin{aligned}
\dot{V}(t)= & \delta(t)^{T}\left(F_{\sigma}^{T} P+P F_{\sigma}\right) \delta(t)+2 \delta(t)^{T} P \Delta(t) \\
& +2 \bar{\Omega}_{0}^{T}(t) \dot{\bar{\Omega}}_{0}(t)+2 \bar{\Omega}^{T}(t) \dot{\bar{\Omega}}(t) \\
= & \delta(t)^{T} Q_{\sigma} \delta(t), \\
Q_{\sigma}= & \left(F_{\sigma}^{T} P+P F_{\sigma}\right) \\
= & {\left[\begin{array}{ccc}
-2 \gamma H_{\sigma} & \gamma I_{n}-\gamma H_{\sigma} & -k H_{\sigma} \\
\gamma I_{n}-\gamma H_{\sigma} & 2 I_{n}(1-l) & 2 l I_{n}-k H_{\sigma} \\
-k H_{\sigma} & 2 l I_{n}-k H_{\sigma} & -2 l I_{n}
\end{array}\right] . }
\end{aligned}
$$

From Lemma 5,

$$
\begin{aligned}
\dot{V}(t) & =\delta(t)^{T} Q_{\sigma} \delta(t)=\sum_{\epsilon=1}^{l_{\sigma}} \delta_{\sigma}^{\epsilon^{T}}(t) Q_{\sigma}^{\epsilon} \delta_{\sigma}^{\epsilon}(t), \\
Q_{\sigma}^{\epsilon} & =\left[\begin{array}{ccc}
-2 \gamma H_{\sigma}^{\epsilon} & \gamma I_{d_{\sigma}^{\epsilon}}-\gamma H_{\sigma}^{\epsilon} & -k H_{\sigma}^{\epsilon} \\
\gamma I_{d_{\sigma}^{\epsilon}}-\gamma H_{\sigma}^{\epsilon} & 2 I_{d_{\sigma}^{\epsilon}}(1-l) & 2 l I_{d_{\sigma}^{\epsilon}}-k H_{\sigma}^{\epsilon} \\
-k H_{\sigma}^{\epsilon} & 2 l I_{d_{\sigma}^{\epsilon}}-k H_{\sigma}^{\epsilon} & -2 l I_{d_{\sigma}^{\epsilon}}
\end{array}\right] .
\end{aligned}
$$

Let

$$
\begin{aligned}
Q_{\sigma}^{\epsilon}= & {\left[\begin{array}{ccc}
-2 \gamma H_{\sigma}^{\epsilon} & \gamma I_{d_{\sigma}^{\epsilon}}-\gamma H_{\sigma}^{\epsilon} & -k H_{\sigma}^{\epsilon} \\
\gamma I_{d_{\sigma}^{\epsilon}}-\gamma H_{\sigma}^{\epsilon} & 2 I_{d_{\sigma}^{\epsilon}}(1-l) & 2 l I_{d_{\sigma}^{\epsilon}}-k H_{\sigma}^{\epsilon} \\
-k H_{\sigma}^{\epsilon} & 2 l I_{d_{\sigma}^{\epsilon}}-k H_{\sigma}^{\epsilon} & -2 l I_{d_{\sigma}^{\epsilon}}
\end{array}\right] } \\
= & {\left[\begin{array}{ccc}
-2 \gamma I_{d_{\sigma}^{\epsilon}} & \gamma H_{\sigma}^{\epsilon^{-1}}-\gamma I_{d_{\sigma}^{\epsilon}} & -k I_{d_{\sigma}^{\epsilon}} \\
\gamma H_{\sigma}^{\epsilon^{-1}}-\gamma I_{d_{\sigma}^{\epsilon}} & 2(1-l) H_{\sigma}^{\epsilon^{-1}} & 2 l H_{\sigma}^{\epsilon^{-1}}-k I_{d_{\sigma}^{\epsilon}} \\
-k I_{d_{\sigma}^{\epsilon}} & 2 l H_{\sigma}^{\epsilon^{-1}}-k I_{d_{\sigma}^{\epsilon}} & -2 l H_{\sigma}^{\epsilon^{-1}}
\end{array}\right] } \\
\widehat{Q}_{\sigma}^{\epsilon}= & {\left[\begin{array}{ccc}
-2 \gamma I_{d_{\sigma}^{\epsilon}} & \gamma H_{\sigma}^{\epsilon^{-1}}-\gamma I_{d_{\sigma}^{\epsilon}} & -k I_{d_{\sigma}^{\epsilon}} \\
\gamma H_{\sigma}^{\epsilon^{-1}}-\gamma I_{d_{\sigma}^{\epsilon}} & 2(1-l) H_{\sigma}^{\epsilon^{-1}} & 2 l H_{\sigma}^{\epsilon^{-1}}-k I_{d_{\sigma}^{\epsilon}} \\
-k I_{d_{\sigma}^{\epsilon}} & 2 l H_{\sigma}^{\epsilon^{-1}}-k I_{d_{\sigma}^{\epsilon}} & -2 l H_{\sigma}^{\epsilon^{-1}}
\end{array}\right] . }
\end{aligned}
$$

From (49), we have

$$
\dot{V}(t) \leq \sum_{\epsilon=1}^{l_{\sigma}} \bar{\lambda}_{\widehat{\mathrm{Q}}_{\sigma}^{\epsilon}} \bar{\lambda}_{H_{\sigma}^{\epsilon}} \delta_{\sigma}^{\epsilon^{T}} \delta_{\sigma}^{\epsilon} \leq \sum_{\epsilon=1}^{l_{\sigma}} \bar{\lambda}_{\widehat{\mathrm{Q}}_{\sigma}^{\epsilon}} \bar{\lambda}_{H_{\sigma}^{\epsilon}}\left\|\delta_{\sigma}^{\epsilon}\right\|^{2} .
$$

$\bar{\lambda}_{\widehat{Q}_{\sigma}^{\epsilon}}, \bar{\lambda}_{H_{\sigma}^{\epsilon}}$ are the maximal eigenvalue of $\widehat{Q}_{\sigma}^{\epsilon}$ and $H_{\sigma}^{\epsilon}$, respectively. 
After calculation, we have $\bar{\lambda}_{\widehat{Q}_{\sigma}^{\epsilon}}<0$, when $k, \gamma$, and $l$ satisfy

$$
\begin{aligned}
k & <2 \sqrt{\frac{4 l \gamma}{\bar{\lambda}_{H_{\sigma}^{e}}^{e}}}, \\
\gamma & >\frac{\sqrt{16 l\left(\bar{\lambda}_{H_{\sigma}^{e}}^{2}-1\right)\left(k^{2} \bar{\lambda}_{H_{\sigma}^{e}}^{4}(l-1)\right)+M^{2}}-M}{8 l\left(\bar{\lambda}_{H_{\sigma}^{e}}^{2}+1\right)}, \\
M & =\bar{\lambda}_{H_{\sigma}^{e}}^{2}\left[3 k^{2}-4 l^{2}-8 k l+\bar{\lambda}_{H_{\sigma}^{e}}\left(4 l^{2}-l+k^{2}+8 k l\right)\right. \\
& \left.-\frac{9}{4} \bar{\lambda}_{H_{\sigma}^{e}}^{2} k^{2}\right],
\end{aligned}
$$

where $\bar{\lambda}_{H_{\sigma}^{e}}^{2}$ is the maximal eigenvalue of $H_{\sigma}^{\epsilon}$.

We know $\underline{\lambda}_{H_{\sigma}^{e}} \geq 0$, so from (52) and Lemma 4, we get

$$
\dot{V}(t) \leq \sum_{\epsilon=1}^{l_{\sigma}} \delta_{\sigma}^{\epsilon^{T}}(t) Q_{\sigma}^{\epsilon} \delta_{\sigma}^{\epsilon}(t) \leq 0 .
$$

Therefore, $\lim _{t \rightarrow \infty} V(t)=V(\infty)$ exists.

Next, we will show that $\lim _{t \rightarrow \infty} \delta(t)=0$. Consider the infinite sequence $\left\{V\left(t_{s}\right) \mid s=0,1, \ldots\right\}$. From the Cauchy convergence criteria, we know, for any $c>0$, there exists a positive integer $N_{c}$ such that $\left|V\left(t_{s+1}\right)-V\left(t_{s}\right)\right|<c, \forall s>N_{c}$. Then we have

$$
\begin{gathered}
\int_{t_{s}}^{t_{s+1}} \dot{V}(t) d t<c \\
\text { or } \int_{t_{s}}^{t_{s+1}} \dot{V}(t) d t>-c .
\end{gathered}
$$

From (53) and Lemma 5, it follows that

$$
\begin{aligned}
\int_{t_{s}}^{t_{s+\tau}} \delta^{T}(t) \delta(t) d t & <\sum_{\epsilon=1}^{l_{\sigma}} \int_{t_{s}}^{t_{s+1}} \delta_{\sigma}^{\epsilon^{T}}(t) \delta_{\sigma}^{\epsilon}(t) d t \\
& <-\sum_{\epsilon=1}^{l_{\sigma}} \frac{c}{\underline{\lambda}_{\widehat{Q}_{\sigma}^{e}} \underline{\lambda}_{H_{\sigma}^{e}}},
\end{aligned}
$$

which implies that

$$
\lim _{t \rightarrow \infty} \int_{t_{s}}^{t_{s+\tau}} \delta^{T}(t) \delta(t)=0 .
$$

Moreover, from the Lyapunov function $V(t)$ and (52), $\delta(t)$, $\bar{\Omega}(t)_{0}$ and $\bar{\Omega}(t)$ are uniformly bounded and $\dot{\delta}(t)$ is also bounded. By the assumption that $\phi_{0}(t)$ and $\phi_{i}(t)$ are uniformly bounded, therefore $\delta^{T}(t) \delta(t)$ is uniformly continuous. Invoking Barbalat's lemma (Lemma 7), we get

$$
\lim _{t \rightarrow \infty} \delta^{T}(t) \delta(t)=0,
$$

which implies that $\lim _{t \rightarrow \infty} \theta(t)=0$ and $\lim _{t \rightarrow \infty} \vartheta(t)=0$; thus the consensus tracking is achieved.
Theorem 9. If the PE condition (61) (which will be mention later) is satisfied and $\dot{\phi}_{i}(i=0,1, \ldots, n)$ are uniformly bounded, by using adaptive law (26), the parameter estimation errors converge to zero.

Before proving the theorem, we introduce the PE condition. We take

$$
\begin{gathered}
\Phi_{r}(t)=\left[\begin{array}{l}
\Phi_{0}(t) \\
\Phi(t)
\end{array}\right], \\
\Phi_{r_{\sigma}}^{\epsilon}(t)=\left[\begin{array}{l}
\Phi_{0_{\sigma}}^{\epsilon}(t) \\
\Phi_{\sigma}^{\epsilon}(t)
\end{array}\right] .
\end{gathered}
$$

The matrix $\Phi_{r}(t)$ is persistently exciting (PE) (Marino and Tomei [17]); that is, there exist two positive real numbers, $T_{0}$ and $\rho$, such that

$$
\begin{aligned}
& \int_{t}^{t+T_{0}} \Phi_{r}(s) \Phi_{r}^{T}(s) d s \geq \rho I_{n}, \\
& \int_{t}^{t+T_{0}} \Phi_{r_{\sigma}}^{\epsilon}(t) \Phi_{r_{\sigma}}^{\epsilon^{T}}(t) d s \geq \underline{\rho}_{n}, \quad \forall \epsilon=1, \ldots, l_{\sigma} .
\end{aligned}
$$

Proof. We prove Theorem 9 by contradiction. Assume that, for any constant $c>0$, there exists $T_{1}>0$; then

$$
\begin{aligned}
\left\|\bar{\Omega}_{0}(t)\right\| & >c, \\
\|\bar{\Omega}(t)\| & >c, \\
\left\|\bar{\Omega}_{0_{\sigma}}^{\epsilon}(t)\right\| & >\underline{c}, \\
\left\|\bar{\Omega}_{\sigma}^{\epsilon}(t)\right\|>\underline{c}, & \\
& \forall t \geq T_{1}, \quad \forall \epsilon=1, \ldots, l_{\sigma} .
\end{aligned}
$$

From Section 2.1, for the infinite sequence of time intervals $\left[t_{r}, t_{r+1}\right), r=0,1, \ldots$, has the subintervals $\left[t_{r_{j}}, t_{r_{j+1}}\right)$, with identical length $t_{\Delta}$; that is, $t_{r_{j+1}}=t_{r_{j}}+t_{\Delta}$.

Define a function

$$
\begin{aligned}
\Psi(t) & =\frac{1}{2}\left[\Omega_{E}^{T}\left(t+t_{\Delta}\right) \Omega_{E}\left(t+t_{\Delta}\right)-\Omega_{E}^{T}(t) \Omega_{E}(t)\right], \\
\Omega_{E}(t) & =\left[\bar{\Omega}_{0}(t), \bar{\Omega}(t)\right]^{T} .
\end{aligned}
$$

And we have

$$
\begin{aligned}
& \Psi_{\sigma}^{\epsilon}(t) \\
& =\frac{1}{2} \sum_{\epsilon=1}^{l_{\sigma}}\left[\Omega_{E_{\sigma}}^{\epsilon^{T}}\left(t+t_{\Delta}\right) \Omega_{E_{\sigma}}^{\epsilon}\left(t+t_{\Delta}\right)-\Omega_{E_{\sigma}}^{\epsilon^{T}}(t) \Omega_{E_{\sigma}}^{\epsilon}(t)\right], \\
& \Omega_{E_{\sigma}}^{\epsilon}(t)=\left[\bar{\Omega}_{0_{\sigma}}^{\epsilon}(t), \bar{\Omega}_{\sigma}^{\epsilon}(t)\right]^{T} .
\end{aligned}
$$

We know $\lim _{t \rightarrow \infty} V(t)=V(\infty)$; from (59) and (46) we have $\lim _{t \rightarrow \infty} \Omega_{E}^{T}(t) \Omega_{E}(t)=V(\infty)$; it shows that

$$
\lim _{t \rightarrow \infty} \Psi(t)=0 .
$$


From (63), for $\forall \alpha>0$, there exists $T_{\alpha}>0$ such that

$$
\left\|\Psi(t)-\Psi\left(t^{\prime}\right)\right\|<\alpha, \quad \forall t, t^{\prime}>T_{\alpha} .
$$

The time derivative of $\Psi(t)$ at time instant $t_{r_{j}}$ is given by

$$
\begin{aligned}
& \dot{\Psi}\left(t_{r_{j}}\right)=\sum_{\epsilon=1}^{l_{\sigma}} \int_{t_{r_{j}}}^{t_{r_{j}}+t_{\Delta}} \frac{d}{d s}\left[\Omega_{E_{\sigma}}^{\epsilon^{T}}(s) \dot{\Omega}_{E_{\sigma}}^{\epsilon}(s)\right] d s \\
& =\sum_{\epsilon=1}^{l_{\sigma}} \int_{t_{r_{j}}}^{t_{r_{j}}+t_{\Delta}} \frac{d}{d s}\left[\Omega_{r_{\sigma}}^{\epsilon^{T}}(s) \Phi_{r_{\sigma}}^{\epsilon}(s)\left(I_{n}+2 I_{n}-I_{n}\right) H_{\sigma}^{\epsilon} \delta_{\sigma}^{\epsilon}(s)\right] d s \\
& =\sum_{\epsilon=1}^{l_{\sigma}} \int_{t_{r_{j}}}^{t_{r_{j}}+t_{\Delta}}\left\{\left[\delta_{\sigma}^{\epsilon^{T}}(s) K_{\sigma}^{\epsilon} H_{\sigma}^{\epsilon} \Phi_{r_{\sigma}}^{\epsilon^{T}}(s) \Phi_{r_{\sigma}}^{\epsilon}(s)-\Omega_{r_{\sigma}}^{\epsilon^{T}}(s) \Phi_{r_{\sigma}}^{\epsilon}(s)\right]\right. \\
& \left.\cdot H_{\sigma}^{\epsilon} K_{\sigma}^{\epsilon}-\Omega_{r_{\sigma}}^{\epsilon^{T}}(s) \Phi_{r_{\sigma}}^{\epsilon}(s) H_{\sigma}^{\epsilon} K_{\sigma}^{\epsilon} E_{\sigma}^{\epsilon}\right\} \delta_{\sigma}^{\epsilon}(s) d s \\
& -2 \sum_{\epsilon=1}^{l_{\sigma}} \int_{t_{r_{j}}}^{t_{r_{j}}+t_{\Delta}} \Omega_{r_{\sigma}}^{\epsilon^{T}}(s) \Phi_{r_{\sigma}}^{\epsilon}(s) H_{\sigma}^{\epsilon^{2}} \Phi_{r_{\sigma}}^{\epsilon^{T}}(s) \Omega_{r_{\sigma}}^{\epsilon^{T}}(s) d s=\xi_{1}-\xi_{2}, \\
& \Omega_{r_{\sigma}}^{\epsilon}(s)=\operatorname{col}\left[-\bar{\Omega}_{0_{\sigma}}^{\epsilon}(t), \bar{\Omega}_{\sigma}^{\epsilon^{T}}(t)\right], \\
& \Phi_{r_{\sigma}}^{\epsilon}(s)=\operatorname{col}\left[\Phi_{0_{\sigma}}^{\epsilon}(t), \Phi_{\sigma}^{\epsilon^{T}}(t)\right], \\
& K_{\sigma}^{\epsilon}=\operatorname{col}[1,2,-1] \otimes I_{d_{\sigma}^{\epsilon}}^{\epsilon},
\end{aligned}
$$

$\xi_{1}$ and $\xi_{2}$ denote the first and the second integrals in the third equality of (67), respectively.

We know $V(t)$ is bounded, so $\delta_{\sigma}^{\epsilon}(t)$ and $\vec{\Omega}_{E_{\sigma}}$ are bounded. Then we can set two constants $Y_{\delta}, Y_{\Omega}>0$, which satisfy $Y_{\delta}>\left\|\delta_{\sigma}^{\epsilon}\right\|$ and $Y_{\Omega}>\left\|\Omega_{E_{\sigma}}^{\epsilon}\right\|$ for all $\epsilon=1, \ldots, l_{\sigma}$.

Meanwhile, $\Phi_{r_{\sigma}}^{\epsilon}(s)$ and $\dot{\Phi}_{r_{\sigma}}^{\epsilon}(s)$ are assumed to be bounded by $Y_{\Phi}$ and $Y_{\dot{\Phi}}$, respectively, for all $\epsilon=1, \ldots, l_{\sigma}$.

Then we have

$$
\xi_{1} \leq \sum_{\epsilon=1}^{l_{\sigma}} Y_{\sigma}^{\epsilon} \int_{t_{r_{j}}}^{t_{r_{j}}+t_{\Delta}}\left\|\delta_{\sigma}^{\epsilon}(s)\right\| d s
$$

where $Y_{\sigma}^{\epsilon}=\sqrt{6} \bar{\lambda}_{H_{\sigma}^{\epsilon}}\left[\sqrt{6} \bar{\lambda}_{H_{\sigma}^{\epsilon}} Y_{\delta} Y_{\Phi}^{2}+Y_{\Omega} Y_{\dot{\Phi}}+\left\|E_{\sigma}^{\epsilon}\right\| Y_{\Omega} Y_{\Phi}\right]$.

$\left\|E_{\sigma}^{\epsilon}\right\|$ denotes the norm bound of $E_{\sigma}^{\epsilon}$, which depends on $\gamma, k, l$, and $\bar{\lambda}_{H_{\sigma}^{\epsilon}}$.

Since $\lim _{t \rightarrow \infty} \delta(t)=0$, we obtain that $\forall \underline{c}>0$; there exists $T_{2}>0$, such that

$$
\xi_{1} \leq \frac{3}{2} \underline{\rho} \underline{\underline{c}} \sum_{\epsilon=1}^{l_{\sigma}} \underline{\lambda}_{H_{\sigma}^{e}}^{2}, \quad \forall t_{r_{j}} \geq T_{2}
$$

Now we consider $\xi_{2}$. From the assumption (62), we have $\left\|\Omega_{r}(t)\right\|>c$ and $\left\|\Omega_{r_{\sigma}}^{\epsilon}(t)\right\|>\underline{c}$. From the PE condition (61), we get

$$
\begin{aligned}
\xi_{2} & \geq 2 \sum_{\epsilon=1}^{l_{\sigma}} \underline{\lambda}_{H_{\sigma}^{\epsilon}}^{2} \int_{t_{r_{j}}}^{t_{r_{j}}+t_{\Delta}} \Omega_{r_{\sigma}}^{\epsilon^{T}}(s) \Phi_{r_{\sigma}}^{\epsilon}(s) \Phi_{r_{\sigma}}^{\epsilon^{T}}(s) \Omega_{r_{\sigma}}^{\epsilon^{T}}(s) d s \\
& \geq 2 \sum_{\epsilon=1}^{l_{\sigma}} \underline{\lambda}_{H_{\sigma}^{\epsilon}}^{2}
\end{aligned}
$$

$$
\begin{aligned}
& \cdot \underline{c}^{2} \int_{t_{r_{j}}}^{t_{r_{j}}+t_{\Delta}} \frac{\Omega_{r_{\sigma}}^{\epsilon^{T}}(s)}{\left\|\Omega_{r_{\sigma}}^{\epsilon}(s)\right\|} \Phi_{r_{\sigma}}^{\epsilon}(s) \Phi_{r_{\sigma}}^{\epsilon^{T}}(s) \frac{\Omega_{r_{\sigma}}^{\epsilon}(s)}{\left\|\Omega_{r_{\sigma}}^{\epsilon}(s)\right\|} d s \\
& \geq 2 \underline{\rho} \underline{c^{2}} \sum_{\epsilon=1}^{l_{\sigma}} \underline{\lambda}_{H_{\sigma}^{\epsilon}}^{2}, \quad \forall t_{r_{j}} \geq T_{1} .
\end{aligned}
$$

Thus

$$
\dot{\Psi}\left(t_{r_{j}}\right)=\xi_{1}-\xi_{2} \leq-\frac{1}{2} \underline{\rho} \underline{c}^{2} \sum_{\epsilon=1}^{l_{\sigma}} \underline{\lambda}_{H_{\sigma}^{\epsilon}}^{2}, \quad \forall t_{r_{j}} \geq T_{3},
$$

where $T_{3}=\max \left[T_{1}, T_{\alpha}, T_{2}\right]$.

By the sign-preserving theorem of continuous functions, there exists a time interval $\left[t_{r_{j}}, t_{r_{j}}+\Delta T\right)$ with $t_{r_{j}} \geq T_{3}, \Delta T>0$ such that

$$
\begin{aligned}
& \dot{\Psi}(t)=\xi_{1}-\xi_{2} \leq-\frac{1}{2} \underline{\rho} \underline{c} \sum_{\epsilon=1}^{2} \underline{\lambda}_{H_{\sigma}^{\epsilon}}^{2}, \\
& \forall t \in\left[t_{r_{j}}, t_{r_{j}}+\Delta T\right] .
\end{aligned}
$$

So

$$
\int_{t_{r_{j}}}^{t_{r_{j}}+\Delta T} \dot{\Psi}(t) d t=\frac{1}{2} \underline{\rho} \underline{c}^{2} \Delta T \sum_{\epsilon=1}^{l_{\sigma}} \underline{\lambda}_{H_{\sigma}^{\epsilon}}^{2} .
$$

From Lemma 5, $\sum_{\epsilon=1}^{l_{\sigma}} \underline{\lambda}_{H_{\sigma}^{e}}^{2}>0$, we set $\alpha=$ 2) $\underline{\rho} \underline{c}^{2} \Delta T \sum_{\epsilon=1}^{l_{\sigma}} \underline{\lambda}_{H_{\sigma}^{e}}^{2}$; that is to say,

$$
\Psi\left(t_{r_{j}}\right)-\Psi\left(t_{r_{j}}+\Delta T\right)>\alpha
$$

which contradicts (66). Therefore, conjecture (62) is not true and then the parameter convergence is guaranteed. Hence, the proof is complete.

\section{Simulation Results}

Some numerical simulations will be given in this section to illustrate the results of this paper. Figure 1 shows six different graphs each with six followers (labeled by 1-6) and one leader (labeled by 0 ). The communication topology switches every $0.5 \mathrm{~s}$. We set $l=3$; then by using Theorem 8 , we choose $k=3$, $\gamma=2$, and using the control protocol (21) and adaptive law (26), we obtain the simulation results about tracking error as shown in Figures 2 and 3 and the performance of parameter estimation as shown in Figures 4 and 5, respectively.

Figure 2 shows the trajectories of velocity errors $\left(v_{i}-v_{0}\right)$ between the followers and the leader, and Figure 3 shows the trajectories of position errors $\left(x_{i}-x_{0}\right)$ between the followers and the leader. It is clear that all the line will converge to zero; it means that the tracking errors of velocity and position of each agent will become zero; that is to say all followers can follow the leader.

Figure 4 shows the parameter estimation errors of $\Omega_{0}$ of each agent $\left(\widetilde{\omega}(t)_{0 i}-\omega(t)_{0}\right)$, and Figure 5 shows the parameter 


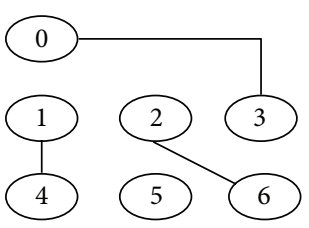

G1

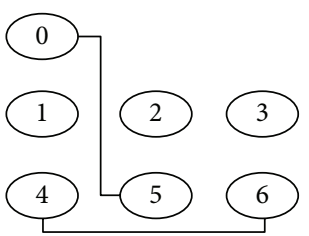

G4

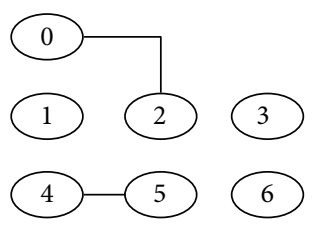

G2

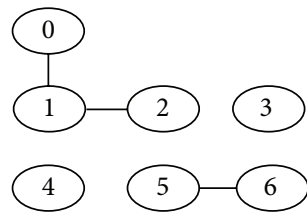

G5

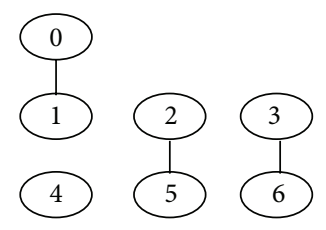

G3

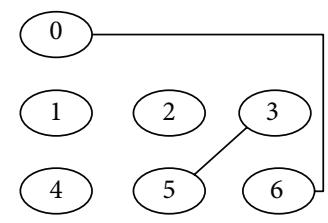

G6

FIGURE 1: Six undirect graphs.

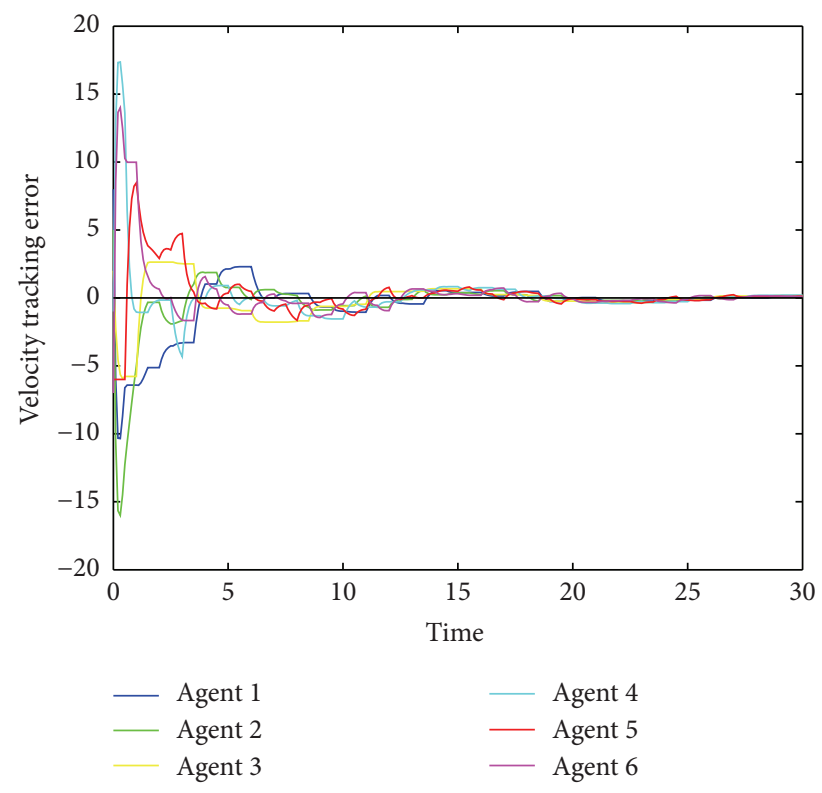

FIGURE 2: The velocity error.

estimation errors of $\Omega_{i}$ of each agent $\left(\widetilde{\omega}(t)_{i}-\omega(t)_{i}\right)$. From the figures, we can ensure that the parameter estimation errors of $\Omega_{0}$ and $\Omega$ can converge to zero; it means the decentralized adaptive laws (26) can estimate the unknown time-varying disturbance $\left(f\left(x_{i}, t\right)=\phi_{i} \omega_{i}\right)$ and the unknown acceleration of the leader $\left(a_{0}(t)=\phi_{o}(t) \omega_{o}\right)$.

\section{Conclusion}

In this paper, we study the adaptive tracking control designed for a second-order leader-following system in jointly connected topology. Moreover, the multiagent system contained unknown disturbance dynamics and the velocity of the leader that is unmeasurable by the followers. To solve such a consensus tracking problem, we proposed a dynamic output-feedback control protocol for tracking the leader

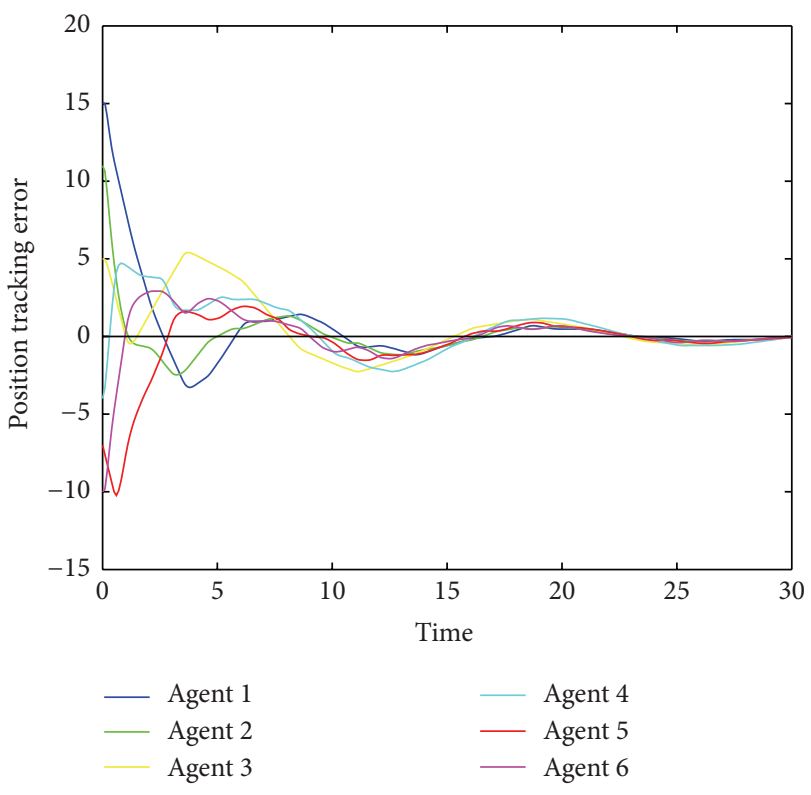

FIgURE 3: The position error.

and employed decentralized adaptive laws to estimate the unknown disturbances. Afterwards, with the help of a common Lyapunov function method and a PE condition, we ensured the tracking stability and the parameter convergence. Finally, simulation results are given to demonstrate the performance of the proposed adaptive tracking control.

\section{Competing Interests}

The authors declare that they have no competing interests.

\section{Acknowledgments}

This work is supported by (1) the Specialized Research Fund for the Doctoral Program of Higher Education of China under Grant no. 20130185110023, (2) National Natural Science 


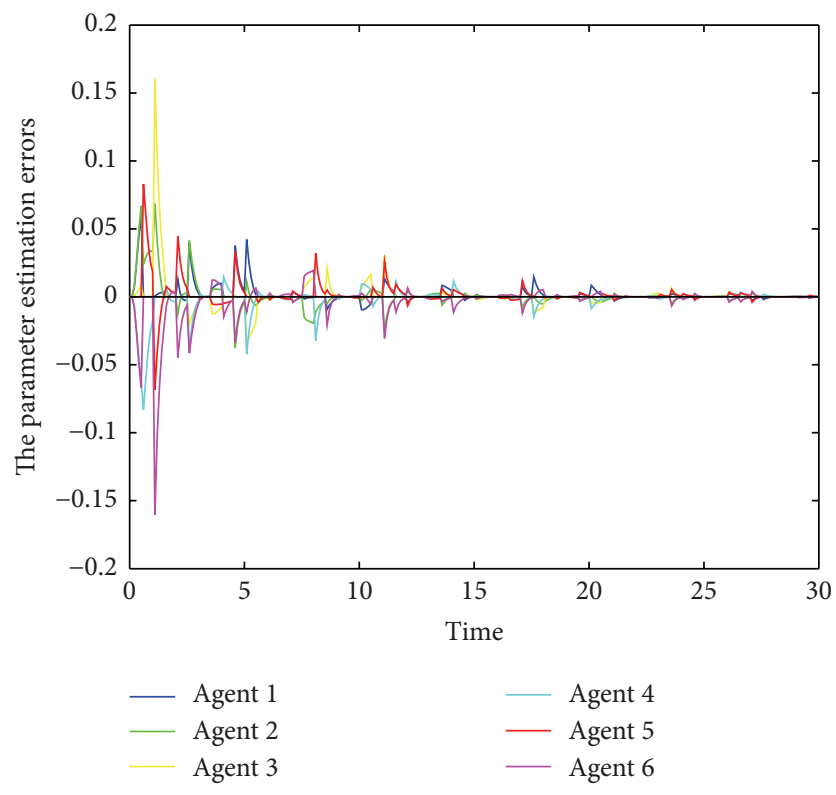

FIgURE 4: The parameter estimation errors of $\omega_{0}$.

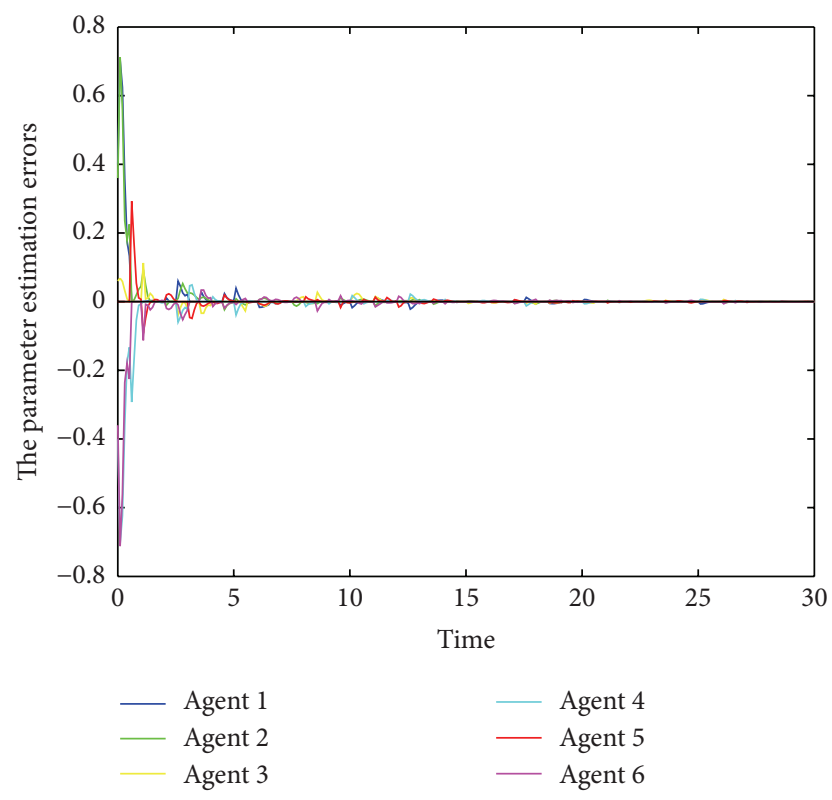

FIGURE 5: The parameter estimation errors of $\omega_{i}$.

Foundation of China under Grants nos. 61104104, 61473061, and 71503206, and (3) the Program for New Century Excellent Talents under Grant no. NCET-13-0091.

\section{References}

[1] W. Ren, "Multi-vehicle consensus with a time-varying reference state," Systems \& Control Letters, vol. 56, no. 7-8, pp. 474-483, 2007.

[2] Y. Hong, J. Hu, and L. Gao, "Tracking control for multiagent consensus with an active leader and variable topology," Automatica, vol. 42, no. 7, pp. 1177-1182, 2006.
[3] J. Hu and Y. Hong, "Leader-following coordination of multiagent systems with coupling time delays," Physica A: Statistical Mechanics and its Applications, vol. 374, no. 2, pp. 853-863, 2007.

[4] K. Peng and Y. Yang, "Leader-following consensus problem with a varying-velocity leader and time-varying delays," Physica A: Statistical Mechanics and its Applications, vol. 388, no. 2-3, pp. 193-208, 2009.

[5] Q. Song, J. Cao, and W. Yu, "Second-order leader-following consensus of nonlinear multi-agent systems via pinning control," Systems \& Control Letters, vol. 59, no. 9, pp. 553-562, 2010.

[6] Y. Hong, G. Chen, and L. Bushnell, "Distributed observers design for leader-following control of multi-agent networks," Automatica, vol. 44, no. 3, pp. 846-850, 2008.

[7] J. Hu, J. Geng, and H. Zhu, "An observer-based consensus tracking control and application to event-triggered tracking," Communications in Nonlinear Science and Numerical Simulation, vol. 20, no. 2, pp. 559-570, 2015.

[8] Y. Zhang and Y. Yang, "Finite-time consensus of secondorder leader-following multi-agent systems without velocity measurements," Physics Letters, Section A: General, Atomic and Solid State Physics, vol. 377, no. 3-4, pp. 243-249, 2013.

[9] D. Bauso, L. Giarré, and R. Pesenti, "Consensus for networks with unknown but bounded disturbances," SIAM Journal on Control and Optimization, vol. 48, no. 3, pp. 1756-1770, 2009.

[10] J. Hu and W. X. Zheng, "Adaptive tracking control of leaderfollower systems with unknown dynamics and partial measurements," Automatica, vol. 50, no. 5, pp. 1416-1423, 2014.

[11] Z. Li, G. Wen, Z. Duan, and W. Ren, "Designing fully distributed consensus protocols for linear multi-agent systems with directed graphs," IEEE Transactions on Automatic Control, vol. 60, no. 4, pp. 1152-1157, 2015.

[12] H. Bai, M. Arcak, and J. T. Wen, "Adaptive design for reference velocity recovery in motion coordination," Systems and Control Letters, vol. 57, no. 8, pp. 602-610, 2008.

[13] Y. Hong, L. Gao, D. Cheng, and J. Hu, "Lyapunov-based approach to multiagent systems with switching jointly connected interconnection," IEEE Transactions on Automatic Control, vol. 52, no. 5, pp. 943-948, 2007.

[14] P. Lin and Y. Jia, "Consensus of a class of second-order multiagent systems with time-delay and jointly-connected topologies," IEEE Transactions on Automatic Control, vol. 55, no. 3, pp. 778-784, 2010.

[15] C. Godsil and G. Royle, Algebraic Graph Theory, vol. 207 of Graduate Texts in Mathematics, Springer, 2001.

[16] P. Lin, K. Qin, H. Zhao, and M. Sun, "A new approach to average consensus problems with multiple time-delays and jointlyconnected topologies," Journal of the Franklin Institute, vol. 349, no. 1, pp. 293-304, 2012.

[17] R. Marino and P. Tomei, Nonlinear Control Design: Geometric, Adaptive and Robust, Prentice Hall, London, UK, 1995.

[18] V.-M. Popov, Hyperstability of Control Systems, Springer, New York, NY, USA, 1973. 


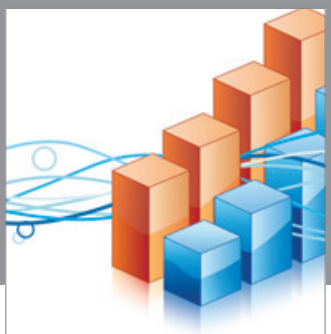

Advances in

Operations Research

vatem alat4

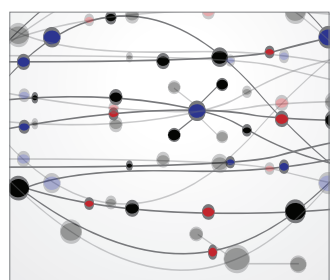

\section{The Scientific} World Journal
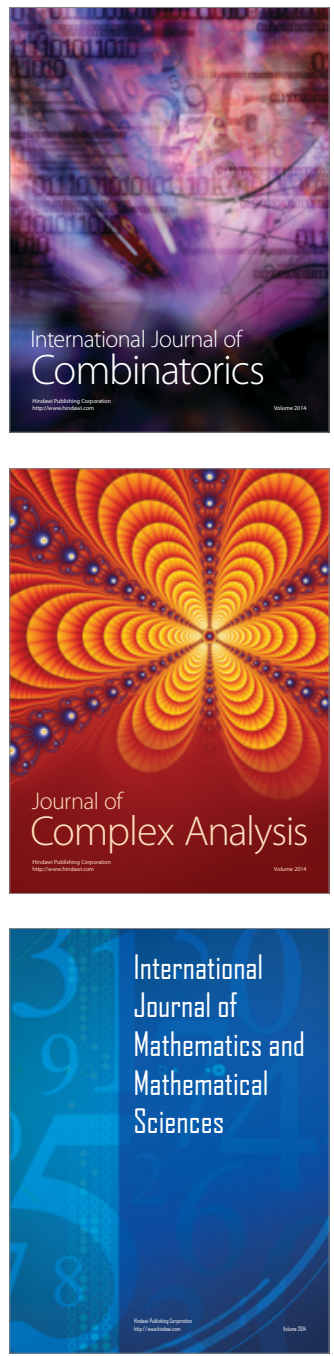
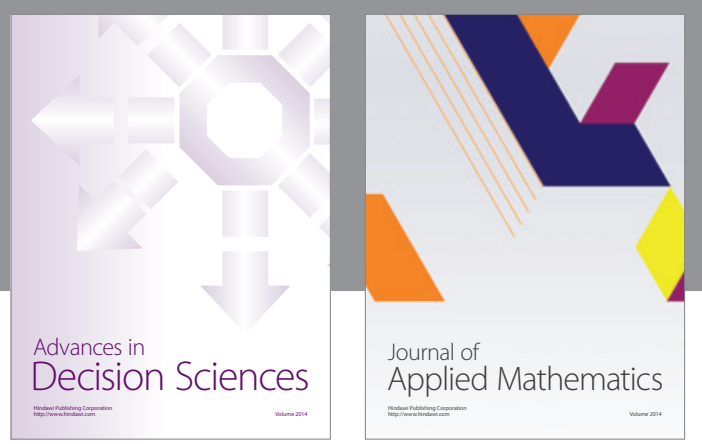

Algebra

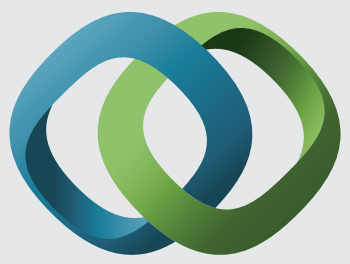

\section{Hindawi}

Submit your manuscripts at

http://www.hindawi.com
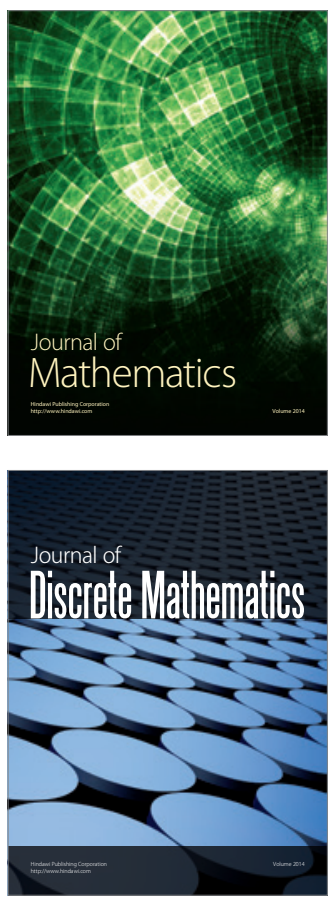

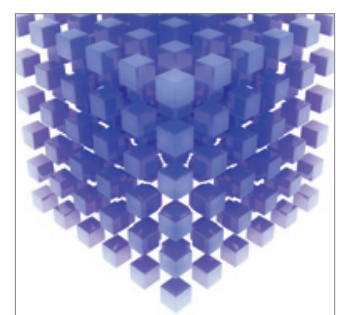

Mathematical Problems in Engineering
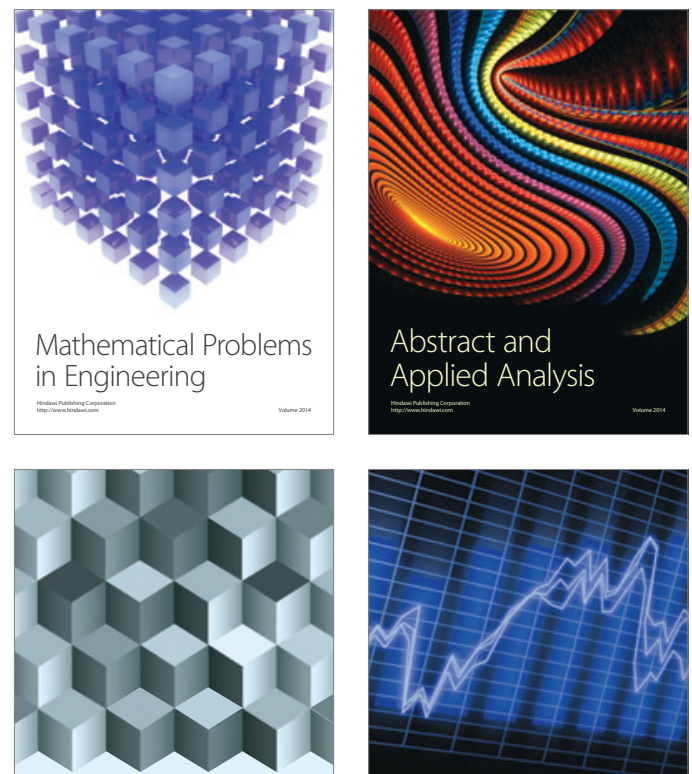

Journal of

Function Spaces

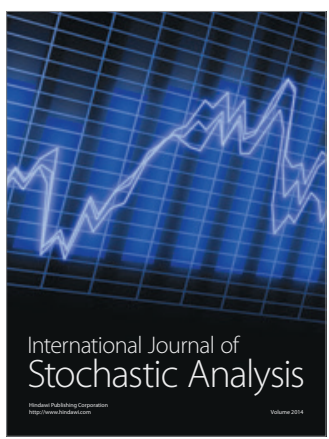

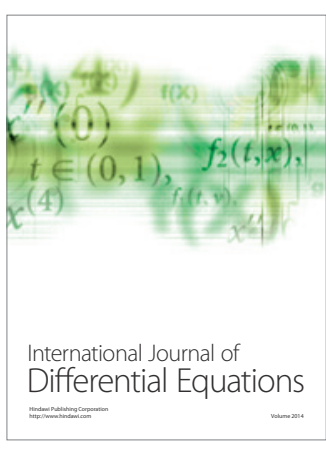
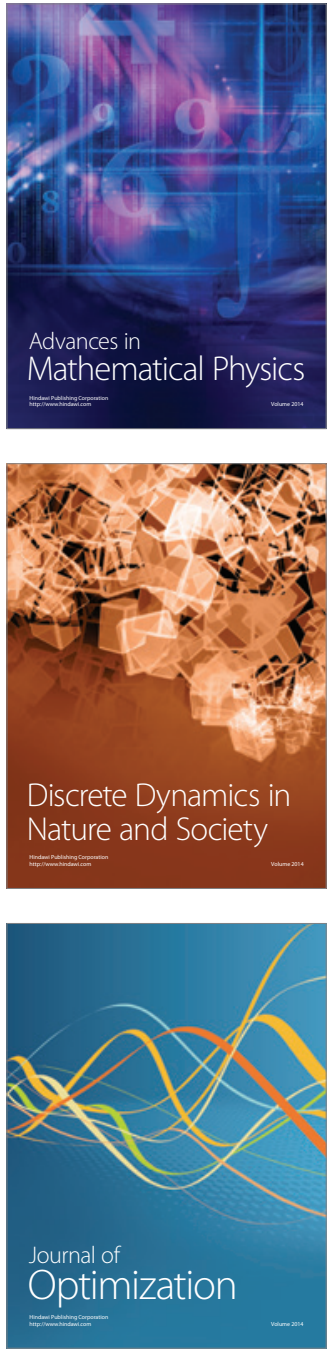